\title{
Peripheral blood neutrophil count as a prognostic factor for patients with hepatocellular carcinoma treated with sorafenib
}

\author{
JIA YUAN ${ }^{1,2}$, HONGMING LIANG ${ }^{1,2}$, JINGHUAN LI $^{1,2}$, MIAO LI $^{1,2}$, BEI TANG $^{1,2}$, \\ HUI MA ${ }^{1,2}$, XIAOYING XIE ${ }^{1,2}$, XIN YIN ${ }^{1,2}$, LAN ZHANG ${ }^{1,2}$ and ZHENGGANG REN ${ }^{1,2}$ \\ ${ }^{1}$ Liver Cancer Institute, Zhongshan Hospital, Fudan University; ${ }^{2}$ Key Laboratory of Carcinogenesis \\ and Cancer Invasion, Ministry of Education, Shanghai 200032, P.R. China
}

Received March 13, 2017; Accepted June 15, 2017

DOI: $10.3892 / \mathrm{mco} .2017 .1416$

\begin{abstract}
Sorafenib is currently the only efficient molecular targeted therapy for hepatocellular carcinoma (HCC), although its effect is relatively moderate and variable between individuals. The present study aimed to evaluate the significance of peripheral blood neutrophils in the prognosis of HCC patients treated with sorafenib. A total of 464 patients with $\mathrm{HCC}$ were treated with sorafenib at Zhongshan Hospital (Shanghai, China) between January 1st, 2008 and December 31st, 2012, among which 120 patients were enrolled in the study. The optimal cutoff point for low vs. high neutrophil count $\left(3.65 \times 10^{9}\right)$ was obtained from a receiver operating characteristic curve. Overall survival (OS) was compared between the patients with low and high peripheral neutrophil counts. Univariate and multivariate analyses were used to explore the prognostic factors associated with OS in the patients treated with sorafenib. A nomogram model was also performed to predict the OS times of these patients. The median OS time was 9.0 months (95\% confidence interval, 5.9-12.1 months) in the whole group of patients, with 1-, 2- and 3-year OS rates of 36, 24 and 16\%, respectively. Using a cutoff level of $3.65 \times 10^{9}$ neutrophils/1, the median OS time was longer in the group of patients with a low peripheral neutrophil count than in those with a high peripheral neutrophil count (11.5 vs. 5.0 months, respectively; $\mathrm{P}<0.001)$. The multivariate analysis showed that peripheral neutrophil count, $\alpha$-fetoprotein level and tumor size were independent prognostic factors for OS. In addition, using the nomogram model for the prediction of OS, the Harrell's c-index was 0.79 . Therefore, it was concluded that a lower peripheral blood neutrophil count was associated with a better prognosis following treatment with sorafenib therapy.
\end{abstract}

Correspondence to: Dr Lan Zhang, Liver Cancer Institute, Zhongshan Hospital, Fudan University, 180 Fenglin Road, Xuhui, Shanghai 200032, P.R. China

E-mail: zhang.lan@zs-hospital.sh.cn

Key words: hepatocellular carcinoma, sorafenib, peripheral neutrophils, prognosis, nomogram

\section{Introduction}

Hepatocellular carcinoma (HCC) is the sixth most prevalent cancer and the second most frequent cause of cancer-associated mortality worldwide, with nearly 780,000 new cases diagnosed annually $(1,2)$. The prognosis of HCC has been improved due to the availability of curative options, such as liver transplantation, hepatic resection and radiofrequency ablation used at an early stage. However, due to its high metastatic potential, more than half of HCC patients develop recurrence or distant metastasis within 5 years post-surgery. Transcatheter arterial chemoembolization (TACE) is the major treatment method for intermediate-stage HCC; however, treatment-refractory disease or metastasis can lead to TACE failure $(3,4)$. Therefore, systemic therapies, such as sorafenib, are applied as alternative treatment options.

Sorafenib is an oral, multi-target and multi-kinase inhibitor, and is used to treat HCC through blocking mitogen-activated protein kinase signaling and through inhibiting vascular endothelial growth factor (VEGF) receptor and platelet-derived growth factor receptor to produce anti-angiogenic effects $(5,6)$. Currently, it is the only molecular targeted drug that exerts a clear survival benefit and has been approved by the Food \& Drug Administration for advanced $\mathrm{HCC}$ (7); it is regarded as a first-line therapy according to the 2008 National Comprehensive Cancer Network guidelines (8). In the SHARP study, the median overall survival (OS) times were 10.7 months in the sorafenib group and 7.9 months in the placebo group $(\mathrm{P}<0.001)$; and the median times to radiological progression were 5.5 months in the sorafenib group and 2.8 months in the placebo group $(\mathrm{P}<0.001)$ (9). The phase III Sorafenib Asia-Pacific trial, restricted to an eastern population, also showed that sorafenib could significantly prolong the median OS time from 4.2 to 6.5 months compared with placebo group (10). However, the objective response rates (ORRs) in these two trials were 2 and $3.3 \%$, suggesting that the efficacy of sorafenib remained limited. A propensity score analysis indicated that initial half-dose sorafenib treatment led to fewer severe adverse effects and a comparable survival benefit compared with a full dose in patients of advanced age (median, 75 years) with HCC (11). In addition, another study indicated that skin toxicity should be closely monitored in HCC patients treated with sorafenib, due to its association with sorafenib efficacy (12). 
To date, the innate mechanisms of tumor pathogenesis and progression remains unclear. However, several studies have indicated that tumor pathogenesis and progression are closely associated with the tumor microenvironment, as well as tumor cells themselves (13). Recent studies have suggested that a systemic inflammatory state is associated with the malignant biological behavior of the tumor $(14,15)$. In particular, the neutrophil-lymphocyte ratio (NLR) has been evaluated as a predictor of prognosis in various types of solid tumor, including gastric, colorectal, pancreatic, breast and lung cancers (16-19). An elevated NLR has already been shown to be associated with poor prognosis in patients with HCC treated by liver transplantation, surgical resection, or TACE (20-22). The present study evaluated the association between peripheral blood neutrophil count and the prognosis of HCC treated with sorafenib, and revealed peripheral neutrophil count as a prognostic factor in such cases.

\section{Patients and methods}

Patients. A total of 464 patients with HCC and who were treated with sorafenib at the Department of Hepatic Oncology of Zhongshan Hospital affiliated to Fudan University (Shanghai, China) between January 1st, 2008 and December 31st, 2012, were initially included in the present study. The study was retrospective and non-interventional. At the time the patients were enrolled, the majority of them had passed away due to disease progression. Therefore, the requirement for informed consent was waived, and the study protocol was approved by the Ethics Committee of Zhongshan Hospital, Fudan University. The study conformed to the principles of the Declaration of Helsinki.

The inclusion criteria were as follows: i) Diagnosis of HCC based on pathology or non-invasive diagnostic criteria [such as liver cirrhosis; evidence of chronic hepatitis B virus (HBV)/hepatitis C virus infection; or dynamic computed tomography (CT) or magnetic resonance imaging (MRI) with intense contrast-uptake during the arterial phase followed by contrast washout during the venous or delayed phases]; and ii) patients received sorafenib therapy continually for $\geq 3$ months. The following exclusion criteria were applied: i) treatment combination included TACE therapy following sorafenib; ii) incomplete clinical parameters were available, affecting outcome analysis; iii) other concurrent cancers were present; iv) leukogenic drug treatment had been taken within 1 week prior to blood collection; v) concurrent infectious diseases were present. Finally, 120 patients were enrolled in the present study, as illustrated in the enrollment flowchart (Fig. 1). The characteristics of these 120 patients are presented in Table I.

Sorafenib treatment. The initial sorafenib oral dose was $400 \mathrm{mg}$ twice daily. Among patients with liver dysfunction of Child-Pugh class B, sorafenib could be initiated at a reduced starting dose of $400 \mathrm{mg}$ once daily, with subsequent dose-escalation according to tolerance. For patients receiving full-dose sorafenib, intake could also be adjusted for the management of adverse events, depending on their type and severity according to the NCI Common Terminology Criteria for Adverse Events (CTCAE) v3.0 (23).
Follow-up and assessment. All patients were followed up every month, which included a routine blood examination, liver function tests and analysis of $\alpha$-fetoprotein (AFP) levels. Response was evaluated by CT or MRI at intervals of 2-3 months. All patients were followed up until December 31st, 2015.

The primary endpoint of the study was OS, which was defined as the time between the commencement of sorafenib therapy and either the date of mortality due to any cause or the last observation date of surviving patients. The secondary endpoint was disease-control rate (DCR), which was defined as the percentage of patients who had a best-response rating of complete response, partial response, or stable disease [according to the Response Evaluation Criteria in Solid Tumors (RECIST) 1.1] (24).

Statistical analysis. Data were evaluated using SPSS software, version 21.0 (IBM Corp., Armonk, NY, USA). Continuous variables were expressed as the mean \pm standard deviation and ranges (minimum-maximum), and were compared using an unpaired Student's t-test, Welch's t-test, or Mann-Whitney rank sum test, according to the normality and homogeneity. Categorical variables were expressed as the frequency and compared using a $\chi^{2}$ test. The optimal cutoff point for neutrophil count was obtained from a receiver operating characteristic (ROC) curve. The Kaplan-Meier method was used to evaluate OS and to carry out the univariate analysis, and the differences between groups were analyzed with a log-rank test. The independent prognostic value of each factor was explored by multivariate analysis according to a Cox proportional hazards model. $\mathrm{P}<0.05$ was considered to indicate a statistically significant difference.

\section{Results}

Patient characteristics. The median age of the 120 enrolled patients was 52.5 years (range, 21-78 years). The majority of the patients were male $(\mathrm{n}=105 ; 87.5 \%)$. HBV surface antigen (HBsAg) was positive in 83 patients (72.2\%). Well-preserved liver function (Child-Pugh class A) was found in 98 patients (89.9\%). Elevated AFP levels (>20 ng/ml) were detected in 87 patients $(72.5 \%)$. There were 57 patients $(52.3 \%)$ who had $\geq 3$ tumors. Tumors measuring $>5 \mathrm{~cm}$ in longest diameter were found in 38 patients (37.3\%). Extrahepatic spread was found in 63 patients $(52.5 \%)$, and vascular invasion in 30 patients (25.0\%). Barcelona Clinic Liver Cancer (BCLC) stage C was found in 79 patients $(65.8 \%)$. The detailed characteristics of the patients are shown in Table I.

OS and objective response. The patients were followed until December 31st, 2015. The median follow-up time was 8.5 months (range, 1.0-80.5 months). At the time of recording of the data, 92 mortalities $(76.7 \%)$ had occurred. The median OS time for the entire cohort was 9.0 months [95\% confidence interval (CI), 5.9-12.1 months], and the 1-, 2- and 3-year OS rates were 36,24 , and $16 \%$, respectively.

The baseline neutrophil count was measured within 1 week prior to sorafenib treatment. The mean baseline level of neutrophils was $3.34 \times 10^{9} / 1$, with a range of $0.7-9.1 \times 10^{9} / 1$. Using an ROC curve, the optimal cutoff point for absolute neutrophil count $\left(3.65 \times 10^{9} / 1\right)$ was determined according to the 
Table I. Baseline demographic and clinical characteristics of the patients $(n=120)$.

\begin{tabular}{|c|c|c|}
\hline Variable & $\mathrm{n}(\%)$ & Average (range) \\
\hline Age (years) & & $52.5(21-78)^{\mathrm{a}}$ \\
\hline$\leq 60$ & $87(72.5)$ & \\
\hline$>60$ & $33(27.5)$ & \\
\hline Sex & & - \\
\hline Male & $105(87.5)$ & \\
\hline Female & $15(12.5)$ & \\
\hline HBsAg status & & - \\
\hline Positive & $83(72.2)$ & \\
\hline Negative & $32(23.4)$ & \\
\hline HBV DNA status & & - \\
\hline Positive & $39(40.6)$ & \\
\hline Negative & $57(59.4)$ & \\
\hline Neutrophil count $\left(\mathrm{x} 10^{9} / 1\right)$ & & $3.34 \pm 1.73(0.7-9.1)^{\mathrm{b}}$ \\
\hline$\leq 3.3$ & $75(62.5)$ & \\
\hline$>3.3$ & $45(37.5)$ & \\
\hline Lymphocyte count $\left(\times 10^{9} / 1\right)$ & & $1.12 \pm 0.51(0.2-2.6)^{\mathrm{b}}$ \\
\hline$\leq 1.1$ & $68(56.7)$ & \\
\hline$>1.1$ & $52(43.3)$ & \\
\hline Platelet count $\left(\times 10^{9} / 1\right)$ & & $\begin{array}{l}121.73 \pm 72.47 \\
(23.0-347.0)^{b}\end{array}$ \\
\hline$<125$ & $77(64.2)$ & \\
\hline$\geq 125$ & $43(35.8)$ & \\
\hline Child-Pugh class & & - \\
\hline $\mathrm{A}$ & $98(89.9)$ & \\
\hline $\mathrm{B}$ & $11(10.1)$ & \\
\hline$\alpha$-fetoprotein level (ng/ml) & & $\begin{array}{c}10,490.31 \pm 19,851.40 \\
(1.2-60,500.0)^{\mathrm{b}}\end{array}$ \\
\hline$\leq 20$ & $28(23.3)$ & \\
\hline$>20$ & $87(72.5)$ & \\
\hline Tumor number & & - \\
\hline$<3$ & $52(47.7)$ & \\
\hline$\geq 3$ & $57(52.3)$ & \\
\hline Tumor size $(\mathrm{cm})$ & & $\begin{array}{l}42.66 \pm 38.99 \\
(0.0-173.0)^{\mathrm{b}}\end{array}$ \\
\hline$\leq 5$ & $64(62.7)$ & \\
\hline$>5$ & $38(37.3)$ & \\
\hline Thrombus & & - \\
\hline Yes & $30(25.0)$ & \\
\hline No & $90(75.0)$ & \\
\hline Metastasis & & - \\
\hline Yes & $63(52.5)$ & \\
\hline No & $57(47.5)$ & \\
\hline BCLC stage & & - \\
\hline $\mathrm{B}$ & $41(34.2)$ & \\
\hline $\mathrm{C}$ & $79(65.8)$ & \\
\hline
\end{tabular}

${ }^{a}$ Median; ${ }^{b}$ mean. Certain data were not available for all patients. HBsAg, HBV surface antigen; HBV, hepatitis B virus; BCLC, Barcelona Clinic Liver Cancer.

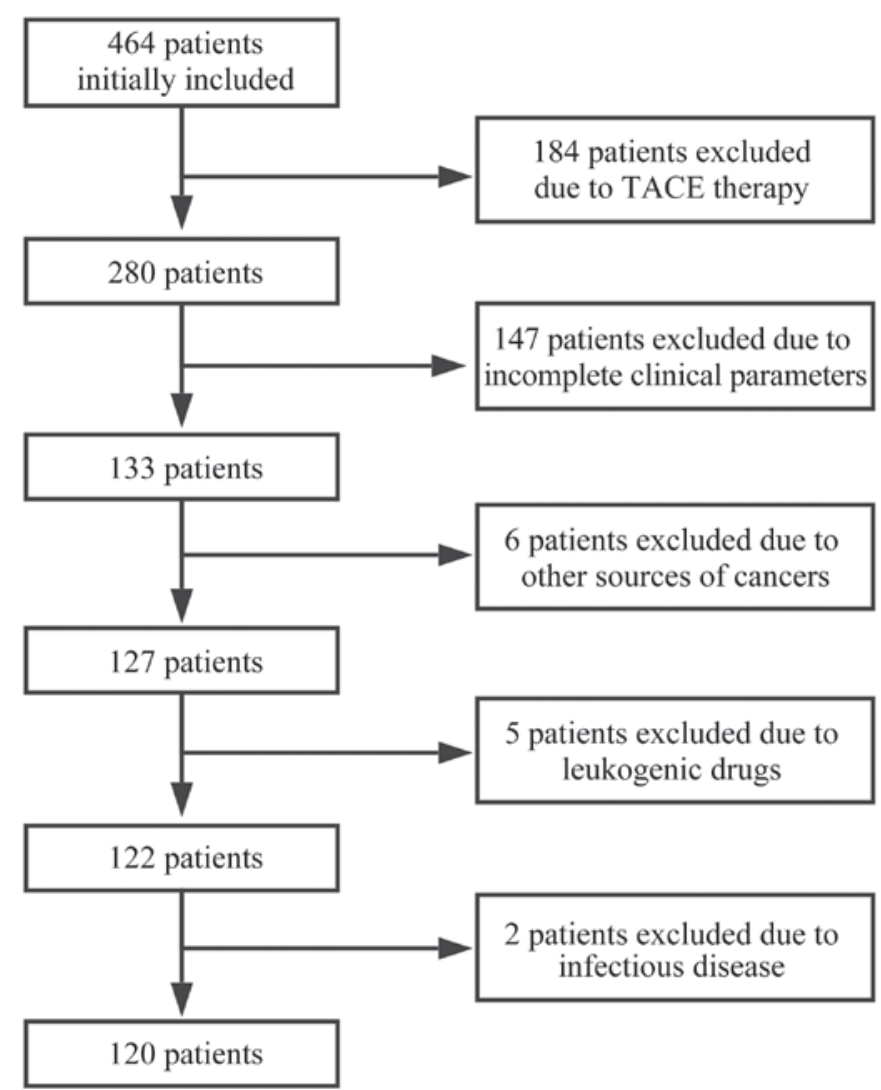

Figure 1. Flow chat illustrating the process of patient enrollment.

median OS time. The area under the curve was 0.612 (95\% CI, $0.504-0.720 ; \mathrm{P}=0.044)$. The sensitivity and specificity were 0.446 , and 0.849 , respectively (Fig. 2A).

According to the cutoff point for neutrophil count determined from the ROC curve, the patients were divided into two groups: A low-neutrophil group (count, $<3.65 \times 10^{9} / 1 ; n=81$ ) and a high-neutrophil group (count, $\geq 3.65 \times 10^{9} / 1 ; n=39$ ). The median OS time of the low-neutrophil group was 11.5 months (95\% CI, 9.7-13.3 months), compared with 5.0 months (95\% CI, 3.2-6.8 months) for patients in the high-neutrophil group $(\mathrm{P}<0.001$; Fig. 2B). In the low-neutrophil group, 2 patients (2.5\%) had a partial response and $34(42.0 \%)$ had stable disease (according to RECIST), whereas in the high-neutrophil group, no patients $(0 \%)$ had a partial response and $8(20.5 \%)$ had stable disease. There were no complete responses in either group. The DCR was significantly higher in the low-neutrophil group than in the high-neutrophil group (44.4 vs. $20.5 \%$; $\mathrm{P}=0.011$; Table II).

Univariate and multivariate analyses of prognostic factors. The potential prognostic factors were evaluated to identify any significant associations with the OS of the patients. As is shown in the Table III, the significant prognostic factors were revealed as $\mathrm{HBsAg}(\mathrm{P}=0.037)$, neutrophil count $(\mathrm{P}<0.001)$, AFP level $(\mathrm{P}<0.001)$, tumor number $(\mathrm{P}=0.001)$, tumor size $(\mathrm{P}<0.001)$, tumor thrombus $(\mathrm{P}=0.005)$, extrahepatic metastasis $(\mathrm{P}=0.030)$ and $\mathrm{BCLC}$ stage $(\mathrm{P}=0.005)$. All the statistically significant prognostic factors in the univariate analysis were included in the multivariate analysis, except BCLC stage (which is evaluated by tumor size, tumor number, tumor thrombus and metastasis). The multivariate analysis identified that a high baseline neutrophil 
Table II. Objective response of patients grouped according to low or high peripheral neutrophil counts.

\begin{tabular}{|c|c|c|c|c|}
\hline \multirow[b]{2}{*}{ Outcome } & \multirow[b]{2}{*}{ Overall } & \multicolumn{2}{|c|}{ Peripheral neutrophils } & \multirow[b]{2}{*}{ P-value } \\
\hline & & Low $(n=81)$ & $\operatorname{High}(n=39)$ & \\
\hline \multicolumn{5}{|l|}{ Level of response, n (\%) } \\
\hline $\mathrm{CR}$ & $0(0.0)$ & $0(0.0)$ & $0(0.0)$ & NA \\
\hline PR & $2(1.7)$ & $2(2.5)$ & $0(0.0)$ & 0.322 \\
\hline SD & $42(35.0)$ & $34(42.0)$ & $8(20.5)$ & $0.021^{\mathrm{a}}$ \\
\hline PD & $44(36.7)$ & $26(32.1)$ & $18(46.2)$ & 0.135 \\
\hline NA & $32(26.7)$ & $19(23.5)$ & $13(33.3)$ & 0.252 \\
\hline Disease-control rate, $\%$ & 36.7 & 44.4 & 20.5 & $0.011^{\mathrm{a}}$ \\
\hline
\end{tabular}

${ }^{\text {aS }}$ tatistically significant $(\mathrm{P}<0.05)$. Low, $\leq 3.65 \times 10^{9}$ neutrophils/l; high, $>3.65 \times 10^{9}$ neutrophils/1; CR, complete remission; $\mathrm{PR}$, partial remission; $\mathrm{SD}$, stable disease; $\mathrm{PD}$, progressive disease; NA, not applicable.

Table III. Univariate and multivariate analyses of factors in association with overall survival.

\begin{tabular}{|c|c|c|c|c|}
\hline \multirow[b]{2}{*}{ Variable } & \multicolumn{2}{|c|}{ Univariate analysis } & \multicolumn{2}{|c|}{ Multivariate analysis } \\
\hline & $\mathrm{HR}(95 \% \mathrm{CI})$ & P-value & HR $(95 \%$ CI $)$ & P-value \\
\hline Age $(\leq 60$ vs. $>60$ years $)$ & $1.093(0.697-1.714)$ & 0.693 & - & - \\
\hline Sex (male vs. female) & $1.433(0.803-2.556)$ & 0.212 & - & - \\
\hline HBsAg status (negative vs. positive) & $1.656(1.018-2.694)$ & $0.037^{\mathrm{a}}$ & $0.914(0.502-1.662)$ & 0.767 \\
\hline HBV DNA status (negative vs. positive) & $1.306(0.819-2.085)$ & 0.254 & - & - \\
\hline Neutrophil count $\left(\leq 3.65 \times 10^{9}\right.$ vs. $\left.>3.65 \times 10^{9} / 1\right)$ & $2.212(1.422-3.442)$ & $<0.001^{\mathrm{a}}$ & $1.796(1.085-2.973)$ & $0.023^{\mathrm{a}}$ \\
\hline Lymphocyte count $\left(\leq 1.1 \times 10^{9}\right.$ vs. $\left.>1.1 \times 10^{9} / 1\right)$ & $0.816(0.607-1.450)$ & 0.335 & - & - \\
\hline Platelet count $\left(\geq 125 \times 10^{9}\right.$ vs. $\left.<125 \times 10^{9} / 1\right)$ & $0.845(0.543-1.315)$ & 0.447 & - & - \\
\hline Child-Pugh class (A vs. B) & $1.784(0.912-3.491)$ & 0.081 & - & - \\
\hline$\alpha$-fetoprotein level ( $\leq 20 \mathrm{vs} .>20 \mathrm{ng} / \mathrm{ml}$ ) & $3.288(1.875-5.763)$ & $<0.001^{\mathrm{a}}$ & $2.582(1.358-4.910)$ & $0.004^{\mathrm{a}}$ \\
\hline Tumor number (<3 vs. $\geq 3$ ) & $2.116(1.342-3.338)$ & $0.001^{\mathrm{a}}$ & $1.351(0.820-2.224)$ & 0.238 \\
\hline Tumor size $(\leq 5$ vs. $>5 \mathrm{~cm})$ & $2.805(1.716-4.584)$ & $<0.001^{\mathrm{a}}$ & $2.387(1.288-4.426)$ & $0.006^{\mathrm{a}}$ \\
\hline Thrombus (yes vs. no) & $1.950(1.203-3.162)$ & $0.005^{\mathrm{a}}$ & $1.021(0.537-1.941)$ & 0.950 \\
\hline Metastasis (yes vs. no) & $1.578(1.036-2.404)$ & $0.030^{\mathrm{a}}$ & $1.222(0.761-1.963)$ & 0.406 \\
\hline BCLC stage (B vs. C) & $1.880(1.190-2.969)$ & $0.005^{\mathrm{a}}$ & - & - \\
\hline
\end{tabular}

${ }^{\text {aS }}$ tatistically significant $(\mathrm{P}<0.05)$. HR, hazard ratio; CI, confidence interval; HBsAg, HBV surface antigen; HBV, hepatitis B virus; -, negative; + , positive; BCLC, Barcelona Clinic Liver Cancer.

count was an independent prognostic factor associated with OS $(\mathrm{P}=0.023, \mathrm{HR}=1.796)$, in addition to AFP level $(\mathrm{P}=0.004)$ and tumor size $(\mathrm{P}=0.006)$ (Table III).

Prognostic nomogram for OS. To predict the 1-year survival and 3-year survival rates for HCC patients treated with sorafenib, a nomogram model that integrated all significant independent factors for OS was produced according to the multivariate Cox regression model (Fig. 3). The Harrell's ç-index for OS prediction was 0.79 .

\section{Discussion}

Currently, sorafenib is the only molecular targeted drug to provide a clear survival benefit for patients with advanced
$\mathrm{HCC}$; it has been shown to prolong the OS time of these patients for $\sim 3$ months (9). A numbers of studies have proven its safety and effectiveness (25). Although the appearance of sorafenib brings hope to patients with advanced HCC, the ORR of sorafenib remains low due to tumor heterogeneity. Therefore, it is important to identify the best prognostic factors that can predict response to sorafenib. Certain studies showed that serum VEGF concentration and extracellular signal-regulated kinase levels were good predictors $(26,27)$; however, other surrogate biomarkers must be explored to evaluate prognosis or the efficacy of treatment with sorafenib HCC.

In the present study, the patients were restricted to those with BCLC stage C or BCLC stage B but who had previously experienced TACE failure, for whom sorafenib is the major treatment method (28). Routine blood tests are a conventional 

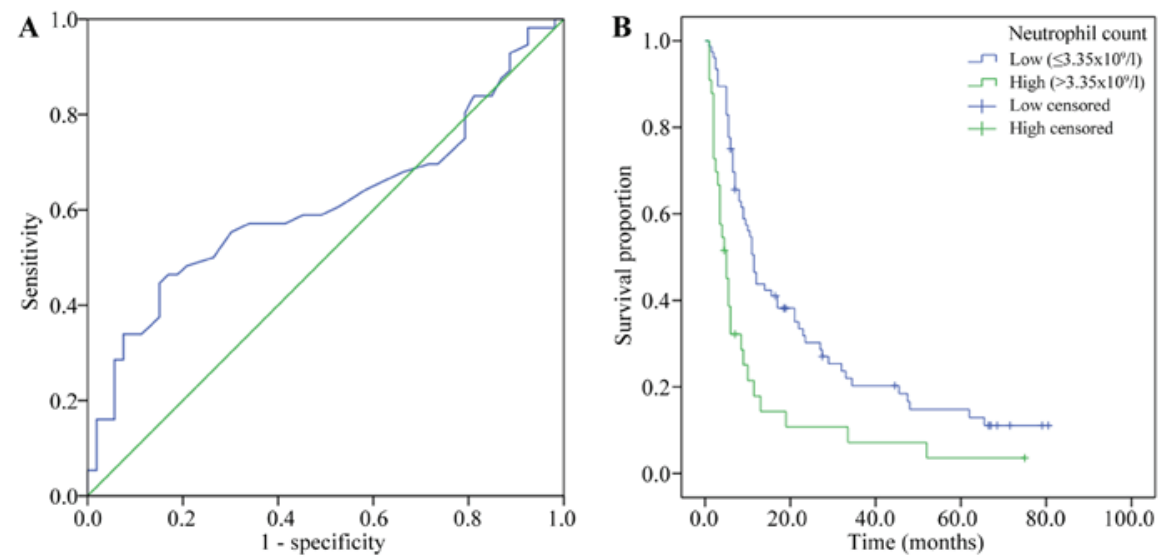

Figure 2. (A) Receiver operating characteristic curve for discrimination of neutrophil counts of HCC patients according to the median OS. (B) Kaplan-Meier survival curves for comparison of OS in the low-neutrophil $\left(<3.65 \times 10^{9} / 1\right)$ and high-neutrophil $\left(\geq 3.65 \times 10^{9} / 1\right)$ groups $(\mathrm{P}<0.001)$.

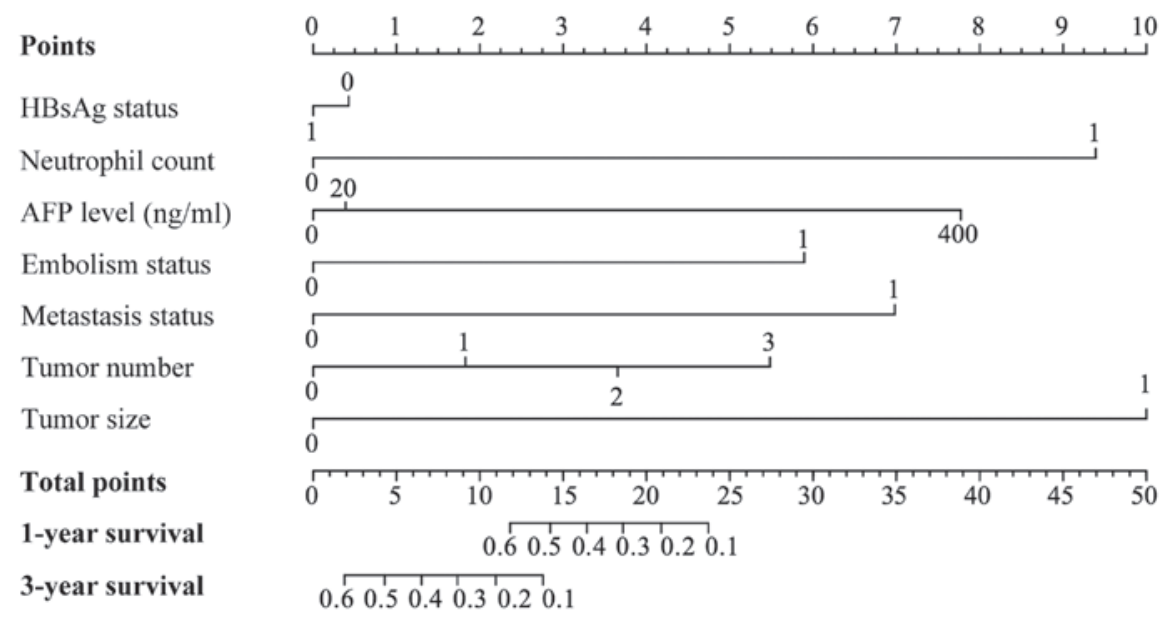

Figure 3. A nomogram predicts the probability of OS based on neutrophil and other prognostic factors in HCC patients treated with sorafenib. To use the nomogram, an individual patient's value is located on each variable axis, and a line is drawn upward to determine the number of points received for each variable value. The sum of these numbers is then located on the 'Total points' axis, and a line is drawn downward to the survival axes to determine the likelihood of survival at 1 or 3 years. The Harrell's c-index for OS prediction was 0.79 . For variables displayed as binary, 0 and 1 correspond to the following values: $\mathrm{HBsAg}$, negative $=0$, positive $=1$; neutrophil count, $\leq 3.65 \times 10^{9} / 1=0,>3.65 \times 10^{9} / 1=1$; thrombus, no $=0$, yes $=1$; metastasis, negative $=0$, positive $=1$; tumor size, $\leq 5 \mathrm{~cm}=0,>5 \mathrm{~cm}=1$. OS, overall survival.

examination for patients, and neutrophil counts are commonly used to evaluate a patient's inflammatory state in clinical practice. Tumor neutrophil infiltration has also been found to be associated with the promotion of inflammation, contributing to tumor progression (29). Therefore, we hypothesized that the peripheral neutrophil count could be a biomarker indicative of the tumor inflammatory microenvironment in HCC and the efficacy of sorafenib treatment. The median OS time of the low-neutrophil group was significantly increased compared with that of the high-neutrophil group (11.5 vs. 5.0 months). In addition, the DCR was significantly higher in the low-neutrophil group than in the high-neutrophil group (44.5 vs. $20.5 \%$ ). Univariate and multivariate analyses revealed that neutrophil level is an independent prognostic factor for OS. In addition, peripheral neutrophil count was associated with platelet count as they were components of the same hematopoietic system. Furthermore, peripheral neutrophil count was associated with AFP level and tumor size (data not shown), suggesting the following possible mechanism: Increased neutrophil levels alter the tumor microenvironment, and the formation of the inflammatory microenvironment affects the sensitivity of HCC to sorafenib treatment, thus promoting tumor growth, resulting in elevated AFP concentration.

In similar studies, researchers have investigated the use of NLR as a prognostic factor. Motomura et al (30) found there was a correlation between elevated NLR and upregulated interleukin (IL)-17 concentration in serum and peritumoral regions. IL-17 is a proinflammatory cytokine that promotes HCC growth (31). In our preliminary data analysis, neutrophil count was found to have a significant association with OS, whereas lymphocyte count did not. In addition, NLR was associated with survival, but was not as significant as the neutrophil count (data not shown). Therefore, in the present research, the absolute neutrophil count was used as a predictor. Furthermore, studies have shown that neutrophils are associated with the systemic release of growth factors and proteolytic enzymes, such as VEGF and matrix metalloproteinase-9, which promote tumor invasion, metastasis and angiogenesis $(32,33)$.

In present study, a nomogram model was created to predict the survival rate based on neutrophil count and other prognostic 
factors. We suggest that this model may be a simple and easy tool for estimating the survival probability of patients with HCC. The Harrell's c-index was 0.79 for this model, indicating that this model was basically consistent with the actual conditions.

Although the present study has revealed a relationship between peripheral neutrophil count and the efficacy of sorafenib treatment, the underlying mechanisms require further exploration. As a retrospective study, there are certain limitations, such as the presence of possible bias, and further prospective randomized controlled trials are encouraged to evaluate the predictive role of peripheral neutrophils in the efficacy of sorafenib treatment for HCC.

\section{References}

1. Bruix J, Gores GJ and Mazzaferro V: Hepatocellular carcinoma: Clinical frontiers and perspectives. Gut 63: 844-855, 2014.

2. Abdel-Rahman O: Systemic therapy for hepatocellular carcinoma (HCC): From bench to bedside. J Egypt Natl Canc Inst 25: 165-171, 2013.

3. Llovet JM and Bruix J: Novel advancements in the management of hepatocellular carcinoma in 2008. J Hepatol 48 (Suppl 1): S20-S37, 2008.

4. Llovet JM, Burroughs A and Bruix J: Hepatocellular carcinoma. Lancet 362: 1907-1917, 2003.

5. Chen W, Wu J, Shi H, Wang Z, Zhang G, Cao Y, Jiang C and Ding Y: Hepatic stellate cell coculture enables sorafenib resistance in Huh7 cells through HGF/c-Met/Akt and Jak2/Stat3 pathways. Biomed Res Int 2014: 764981, 2014.

6. Moeini A, Cornellà H and Villanueva A: Emerging signaling pathways in hepatocellular carcinoma. Liver Cancer 1: 83-93, 2012.

7. Huang WS and Yang CH: Sorafenib induced tumor lysis syndrome in an advanced hepatocellular carcinoma patient. World J Gastroenterol 15: 4464-4466, 2009.

8. National Comprehensive Cancer Network. (NCCN) Clinical Practice Guidelines in Oncology. Hepatobiliary Cancers, 2008. https: //www.nccn.org/professionals/ physician_gls/f_guidelines. asp. Accessed February 8, 2008.

9. Llovet JM, Ricci S, Mazzaferro V, Hilgard P, Gane E, Blanc JF, de Oliveira AC, Santoro A, Raoul JL, Forner A, et al: Sorafenib in advanced hepatocellular carcinoma. N Engl J Med 359: 378-390, 2008

10. Cheng AL, Guan Z, Chen Z, Tsao CJ, Qin S, Kim JS, Yang TS, Tak WY, Pan H, Yu S, et al: Efficacy and safety of sorafenib in patients with advanced hepatocellular carcinoma according to baseline status: Subset analyses of the phase III Sorafenib Asia-Pacific trial. Eur J Cancer 48: 1452-1465, 2012.

11. Morimoto M, Numata K, Kondo M, Kobayashi S, Ohkawa S, Hidaka H, Nakazawa T, Okuwaki Y, Okuse C, Matsunaga K, et al: Field practice study of half-dose sorafenib treatment on safety and efficacy for hepatocellular carcinoma: A propensity score analysis. Hepatol Res 45: 279-287, 2015.

12. Vincenzi B, Santini D, Russo A, Addeo R, Giuliani F, Montella L, Rizzo S, Venditti O, Frezza AM, Caraglia M, et al: Early skin toxicity as a predictive factor for tumor control in hepatocellular carcinoma patients treated with sorafenib. Oncologist 15: 85-92, 2010.

13. Mbeunkui F and Johann DJ Jr: Cancer and the tumor microenvironment: A review of an essential relationship. Cancer Chemother Pharmacol 63: 571-582, 2009.

14. Li C, Deng M, Hu J, Li X, Chen L, Ju Y, Hao J and Meng S: Chronic inflammation contributes to the development of hepatocellular carcinoma by decreasing miR-122 levels. Oncotarget 7: 17021-17034, 2016.

15. Chang TS, Chen CL, Wu YC, Liu JJ, Kuo YC, Lee KF, Lin SY, Lin SE, Tung SY, Kuo LM, et al: Inflammation promotes expression of stemness-related properties in HBV-related hepatocellular carcinoma. PLoS One 11: e149897, 2016.

16. Wang F, Liu ZY, Xia YY, Zhou C, Shen XM, Li XL, Han SG, Zheng Y, Mao ZQ, Gong FR, et al: Changes in neutrophil/lymphocyte and platelet/lymphocyte ratios after chemotherapy correlate with chemotherapy response and prediction of prognosis in patients with unresectable gastric cancer. Oncol Lett 10: 3411-3418, 2015.
17. Pine JK, Morris E, Hutchins GG, West NP, Jayne DG, Quirke P and Prasad KR: Systemic neutrophil-to-lymphocyte ratio in colorectal cancer: The relationship to patient survival, tumour biology and local lymphocytic response to tumour. Br J Cancer 113: 204-211, 2015.

18. Szkandera J, Stotz M, Eisner F, Absenger G, Stojakovic T, Samonigg H, Kornprat P, Schaberl-Moser R, Alzoughbi W, Ress AL, et al: External validation of the derived neutrophil to lymphocyte ratio as a prognostic marker on a large cohort of pancreatic cancer patients. PLoS One 8: e78225, 2013

19. Shao N and Cai Q: High pretreatment neutrophil-lymphocyte ratio predicts recurrence and poor prognosis for combined small cell lung cancer. Clin Transl Oncol 17: 772-778, 2015.

20. Xiao GQ, Liu C, Liu DL, Yang JY and Yan LN: Neutrophil-lymphocyte ratio predicts the prognosis of patients with hepatocellular carcinoma after liver transplantation. World J Gastroenterol 19: 8398-8407, 2013.

21. Okamura Y, Ashida R, Ito T, Sugiura T, Mori K and Uesaka K: Preoperative neutrophil to lymphocyte ratio and prognostic nutritional index predict overall survival after hepatectomy for hepatocellular carcinoma. World J Surg 39: 1501-1509, 2015.

22. Fan W, Zhang Y, Wang Y, Yao X, Yang J and Li J: Neutrophil-to-lymphocyte and platelet-to-lymphocyte ratios as predictors of survival and metastasis for recurrent hepatocellular carcinoma after transarterial chemoembolization. PLoS One 10: e119312, 2015.

23. Trotti A, Colevas AD, Setser A, Rusch V, Jaques D, Budach V, Langer C, Murphy B, Cumberlin R, Coleman CN and Rubin P: CTCAE v3.0: Development of a comprehensive grading system for the adverse effects of cancer treatment. Semin Radiat Oncol 133: 176-181, 2003.

24. Therasse P, Arbuck SG, Eisenhauer EA, Wanders J, Kaplan RS, Rubinstein L, Verweij J, Van Glabbeke M, van Oosterom AT, Christian MC and Gwyther SG: New guidelines to evaluate the response to treatment in solid tumors. European Organization for Research and Treatment of Cancer, National Cancer Institute of the United States, National Cancer Institute of Canada. J Natl Cancer Inst 92: 205-216, 2000.

25. Ye SL, Chen X, Yang J, Bie P, Zhang S, Liu F, Liu L, Zhou J, Dou K, Hao C, et al: Safety and efficacy of sorafenib therapy in patients with hepatocellular carcinoma: Final outcome from the Chinese patient subset of the GIDEON study. Oncotarget 7: 6639-6648, 2016.

26. Llovet JM, Peña CE, Lathia CD, Shan M, Meinhardt G and Bruix J; SHARP Investigators Study Group: Plasma biomarkers as predictors of outcome in patients with advanced hepatocellular carcinoma. Clin Cancer Res 18: 2290-2300, 2012.

27. Zhang Z, Zhou X, Shen H, Wang D and Wang Y: Phosphorylated ERK is a potential predictor of sensitivity to sorafenib when treating hepatocellular carcinoma: Evidence from an in vitro study. BMC Med 7: 41, 2009.

28. Bolondi L, Burroughs A, Dufour JF, Galle PR, Mazzaferro V, Piscaglia F, Raoul JL and Sangro B: Heterogeneity of patients with intermediate (BCLC B) Hepatocellular Carcinoma: Proposal for a subclassification to facilitate treatment decisions. Semin Liver Dis 32: 348-359, 2012.

29. Kim J and Bae JS: Tumor-associated macrophages and neutrophils in tumor microenvironment. Mediators Inflamm 2016: 6058147, 2016.

30. Motomura T, Shirabe K, Mano Y, Muto J, Toshima T, Umemoto Y, Fukuhara T, Uchiyama H, Ikegami T, Yoshizumi T, et al: Neutrophil-lymphocyte ratio reflects hepatocellular carcinoma recurrence after liver transplantation via inflammatory microenvironment. J Hepatol 58: 58-64, 2013.

31. Kuang DM, Zhao Q, Wu Y, Peng C, Wang J, Xu Z, Yin XY and Zheng L: Peritumoral neutrophils link inflammatory response to disease progression by fostering angiogenesis in hepatocellular carcinoma. J Hepatol 54: 948-955, 2011.

32. Kusumanto YH,Dam WA, Hospers GA, Meijer C and Mulder NH: Platelets and granulocytes, in particular the neutrophils, form important compartments for circulating vascular endothelial growth factor. Angiogenesis 6: 283-287, 2003.

33. Li XF, Chen DP, Ouyang FZ, Chen MM, Wu Y, Kuang DM and Zheng L: Increased autophagy sustains the survival and pro-tumourigenic effects of neutrophils in human hepatocellular carcinoma. J Hepatol 62: 131-139, 2015. 